\title{
Transition temperature of a dilute homogeneous imperfect Bose gas
}

\author{
Peter Arnold \\ Department of Physics, University of Virginia, P.O. Box 400714, Charlottesville, VA 22904-4714 \\ Guy Moore \\ Department of Physics, University of Washington, Seattle, Washington 98195-1560
}

(August 27, 2018)

\begin{abstract}
The leading-order effect of interactions on a homogeneous Bose gas is theoretically predicted to shift the critical temperature by an amount $\Delta T_{\mathrm{c}} \simeq \# a_{\mathrm{sc}} n^{1 / 3} T_{0}$ from the ideal gas result $T_{0}$, where $a_{\mathrm{sc}}$ is the scattering length and $n$ is the density. There have been several different theoretical estimates for the numerical coefficient \#. We claim to settle the issue by measuring the numerical coefficient in a lattice simulation of $\mathrm{O}(2) \phi^{4}$ field theory in three dimensions - an effective theory which, as observed previously in the literature, can be systematically matched to the dilute Bose gas problem to reproduce non-universal quantities such as the critical temperature. We find \# $=1.32 \pm 0.02$.
\end{abstract}

The phase transition temperature $T_{0}$ of an ideal threedimensional Bose-Einstein gas at fixed density is something that every physicist learns to calculate in graduate school, if not before. It is amusing that the first correction to that result, from arbitrarily weak interactions, is sufficiently challenging that there has not yet been theoretical agreement on its magnitude. It is understood that, in the weak interaction limit, the correction $\Delta T_{\mathrm{c}} \equiv T_{\mathrm{c}}-T_{0}$ behaves parametrically as

$$
\frac{\Delta T_{\mathrm{c}}}{T_{0}} \rightarrow c a_{\mathrm{sc}} n^{1 / 3}
$$

where $c$ is a numerical coefficient. (A clean argument may be found in Ref. [1].) Here $a_{\mathrm{sc}}$ is the scattering length, and the weak interaction (or dilute) limit is $a_{\mathrm{sc}} n^{1 / 3} \ll 1$ : that is, $a_{\mathrm{sc}}$ is small compared to the typical separation between particles. (We will assume that the interaction is repulsive.) However, there has been little agreement in estimates of the coefficient $c$, a variety of which [1 9] are shown in Fig. 1. Some of these estimates are advertised as rough, but others are not. The difficulty arises because the phase transition is second order, and perturbation theory typically breaks down at second-order phase transitions: the physics that determines $\Delta T_{\mathrm{c}}$ is non-perturbative. As we shall briefly review, the problem of finding $\Delta T_{\mathrm{c}}$ in the weak interaction limit can be related to solving static three-dimensional $\mathrm{O}(2)$ scalar $\phi^{4}$ field theory [1]. In this paper, we present results from using standard, numerical, lattice Monte Carlo methods to solve that theory. In principle this provides an exact method for computing $c$ to any desired precision; in practice, one is limited by computer time and memory. Working on desktop computers, we find $c=1.32 \pm 0.02$, which is the grey bar in Fig. 1.

It is long distance physics that determines $\Delta T_{\mathrm{c}}$ in the weak interaction limit. It is well known 10 that, at distance scales large compared to the scattering length $a_{\mathrm{sc}}$, an appropriate effective theory for a dilute Bose gas is the second-quantized Schrödinger equation, together with a

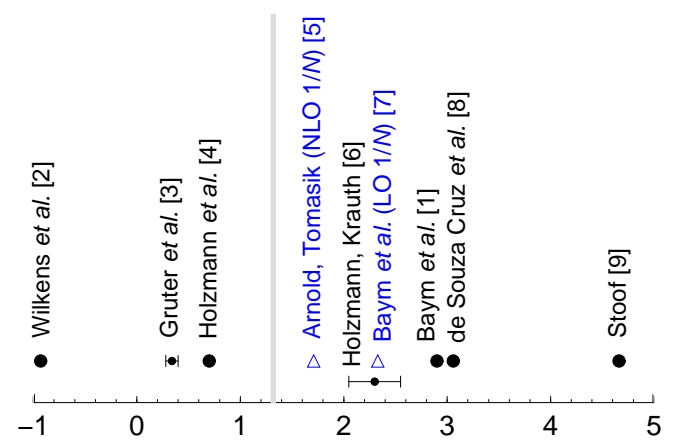

FIG. 1. Estimates from the literature of the constant $c$ in $\Delta T_{\mathrm{c}} / T_{0} \rightarrow \mathrm{can}^{1 / 3}$. The grey bar is the result of this paper.

chemical potential $\mu$ that couples to particle number density $\psi^{*} \psi$, and a $|\psi|^{4}$ contact interaction that reproduces low-energy scattering. The corresponding Lagrangian is

$$
\mathcal{L}=\psi^{*}\left(i \hbar \partial_{t}+\frac{\hbar^{2}}{2 m} \nabla^{2}+\mu\right) \psi-\frac{2 \pi \hbar^{2} a_{\mathrm{sc}}}{m}\left(\psi^{*} \psi\right)^{2} .
$$

Corrections to this effective theory (due, for instance, to energy dependence of the cross-section or 3-body interactions) may be ignored for the propose of computing the leading-order result for $\Delta T_{\mathrm{c}}$. To study (2) at finite temperature, apply the imaginary time formalism, so that $t$ becomes $i \tau$ and imaginary time $\tau$ is periodic with period $\hbar \beta=\hbar / k_{\mathrm{B}} T$. The field $\psi$ can then be decomposed into frequency modes with Matsubara frequencies $\omega_{n}=2 \pi n / \hbar \beta$. For distances large compared to the thermal wavelength $\lambda=\hbar \sqrt{2 \pi \beta / m}$, and sufficiently near the transition so that $|\mu| \ll T$, the non-zero Matsubara frequencies decouple from the dynamics, leaving behind an effective theory of only the zero-frequency modes $\psi_{0}$, with the action $S=\hbar^{-1} \int_{0}^{\hbar \beta} d \tau \int d^{3} x \mathcal{L}$ becoming [1]

$\beta \int d^{3} x\left[\psi_{0}^{*}\left(-\frac{\hbar^{2}}{2 m} \nabla^{2}-\mu_{\mathrm{eff}}\right) \psi_{0}+\frac{2 \pi \hbar^{2} a_{\mathrm{sc}}}{m}\left(\psi_{0}^{*} \psi_{0}\right)^{2}\right]$, 
up to corrections that again do not affect the leadingorder result for $\Delta T_{\mathrm{c}}$. This action can be thought of as the $\beta H$ of a classical three-dimensional field theory. Finally, it is convenient to rewrite $\psi_{0}=\left(\phi_{1}+i \phi_{2}\right) \sqrt{2 \pi} / \lambda$ so that the effective action becomes a conventionally normalized $\mathrm{O}(2)$ field theory:

$$
S=\int d^{3} x\left[\frac{1}{2}|\nabla \phi|^{2}+\frac{1}{2} r \phi^{2}+\frac{u}{4 !}\left(\phi^{2}\right)^{2}\right],
$$

where $\phi$ is understood to be a 2-component real vector $\left(\phi_{1}, \phi_{2}\right)$ and $u=96 \pi^{2} a_{\mathrm{sc}} / \lambda^{2}$.

As noted by Baym et al. [1], it is technically somewhat more convenient, in this formalism, to calculate the shift $\Delta n_{\mathrm{c}}(T)$ in the critical density at fixed temperature instead of the shift $\Delta T_{\mathrm{c}}(n)$ in the critical temperature at fixed density. The two are trivially related at first order in $\Delta T_{\mathrm{c}}$ by $\Delta T_{\mathrm{c}} / T=-\frac{2}{3} \Delta n_{\mathrm{c}} / n$, where the factor of $\frac{2}{3}$ arises from the ideal gas relation $T_{0} \propto n^{2 / 3}$. In the field theory, $n$ is given by $\left\langle\psi^{*} \psi\right\rangle$, which is proportional to $\left\langle\phi^{2}\right\rangle$. Putting everything together [1],

$$
\frac{\Delta T_{\mathrm{c}}}{T_{0}}=-\frac{2 m k_{\mathrm{B}} T_{0}}{3 \hbar^{2} n} \Delta\left\langle\phi^{2}\right\rangle_{\mathrm{c}},
$$

where

$$
\Delta\left\langle\phi^{2}\right\rangle_{\mathrm{c}} \equiv\left[\left\langle\phi^{2}\right\rangle_{\mathrm{c}}\right]_{u}-\left[\left\langle\phi^{2}\right\rangle_{\mathrm{c}}\right]_{0}
$$

is the difference between the effective theory value of $\left\langle\phi^{2}\right\rangle$, at the critical point, for the cases of (i) $u$ small and (ii) the ideal gas $u=0$. Unlike $\left\langle\phi^{2}\right\rangle_{\mathrm{c}}$, the difference $\Delta\left\langle\phi^{2}\right\rangle_{\mathrm{c}}$ is an infrared quantity, independent of how the effective theory (4) is regularized in the ultraviolet (UV).

Finding $\left[\left\langle\phi^{2}\right\rangle_{\mathrm{c}}\right]_{u}$ in the effective theory (4) corresponds to fixing $u$, varying $r$ to reach the critical point, and then measuring $\left\langle\phi^{2}\right\rangle$. The only parameter of this problem is $u$. By dimensional analysis, physics is therefore non-perturbative at the infrared length scale $1 / u$, and, again by dimensional analysis, $\Delta\left\langle\phi^{2}\right\rangle_{\mathrm{c}}$ is proportional to $u$. Putting this together with the ideal gas formula $T_{0}=\left(2 \pi \hbar^{2} / k_{\mathrm{B}} m\right)\left[n / \zeta\left(\frac{3}{2}\right)\right]^{2 / 3}$, one may summarize the relationship between the weak interaction limit for $\Delta T_{\mathrm{c}}$ and the $\mathrm{O}(2)$ effective theory as $\Delta T_{\mathrm{c}} / T_{0} \rightarrow c a_{\mathrm{sc}} n^{1 / 3}$ with

$$
c=-\frac{128 \pi^{3}}{\left[\zeta\left(\frac{3}{2}\right)\right]^{4 / 3}} \frac{\Delta\left\langle\phi^{2}\right\rangle_{\mathrm{c}}}{u} .
$$

To compute $\Delta\left\langle\phi^{2}\right\rangle_{\mathrm{c}} / u$, we put the $\mathrm{O}(2)$ theory (4) on a lattice. For example, the most straightforward discretization would use the action

$$
\begin{aligned}
S_{\mathrm{U}}= & a_{\text {lat }}^{3} \sum_{\langle\mathbf{x y}\rangle} \frac{1}{2}\left[\left(\frac{\phi_{1 \mathbf{x}}-\phi_{1 \mathbf{y}}}{a_{\text {lat }}}\right)^{2}+\left(\frac{\phi_{2 \mathbf{x}}-\phi_{2 \mathbf{y}}}{a_{\text {lat }}}\right)^{2}\right] \\
& +a_{\text {lat }}^{3} \sum_{\mathbf{x}}\left[\frac{r_{\text {lat }}}{2}\left(\phi_{1 \mathbf{x}}^{2}+\phi_{2 \mathbf{x}}^{2}\right)+\frac{u}{4 !}\left(\phi_{1 \mathbf{x}}^{2}+\phi_{2 \mathbf{x}}^{2}\right)^{2}\right],
\end{aligned}
$$

on a simple cubic lattice, where $\langle\mathbf{x y}\rangle$ represents all nearest-neighbor pairs and $a_{\text {lat }}$ is the lattice spacing (unrelated to $\left.a_{\mathrm{sc}}\right)$. The dimensionless coupling of the lattice theory is $u a_{\text {lat }}$, and the continuum limit is $u a_{\text {lat }} \rightarrow 0$.

Our simulations use an improved action to reduce lattice spacing errors. (The subscript U on $S$ in Eq. (8) stands for "unimproved.") Details concerning the action and our simulations are given in Ref. [11]. Our simulations use a combination of heat bath and multi-grid updates 12. At finite volume, we use the method of Binder cumulants 11, 13] to determine a nominal critical value for $r$.

Because three-dimensional scalar theory requires UV regularization of its $\phi^{2}$ interactions, the $r_{\text {lat }}$ in the lattice action (8) is not simply the $r_{\text {eff }}$ in the effective theory action (4), which in turn is not simply related to the chemical potential $\mu$ in the original action (2). However, for our purposes here, we are only interested in adjusting $r_{\text {lat }}$ to find the critical point, for a given $u$, and measuring $\Delta\left\langle\phi^{2}\right\rangle$ there. The actual relation between $r_{\text {lat }}, r_{\text {eff }}$, and $\mu$ is unnecessary [14]. The $\phi^{4}$ interactions, in contrast, do not require UV regularization: in the limit $u a_{\text {lat }} \rightarrow 0$, the coupling $u$ of the lattice theory may be identified with the continuum coupling $u=96 \pi^{2} a_{\mathrm{sc}} / \lambda^{2}$ introduced earlier.

For a given $u$, we compute $\left\langle\phi^{2}\right\rangle_{\mathrm{c}}$ by Monte Carlo numerical simulations of the lattice theory. The $u=0$ piece of the difference $\Delta\left\langle\phi^{2}\right\rangle_{\mathrm{c}}$ can be easily computed without simulations:

$$
\Delta\left\langle\phi^{2}\right\rangle_{\mathrm{c}}=\lim _{a_{\text {lat }} \rightarrow 0}\left[\left\langle\phi^{2}\right\rangle_{\text {lat }}-\int_{\mathbf{p} \in \mathrm{BZ}} G_{\text {lat }}^{i i}(\mathbf{p})\right],
$$

where $G_{\text {lat }}^{i j}(\mathbf{p})$ is the free lattice propagator, and the momentum $\mathbf{p}$ is integrated over the Brillouin zone (BZ). Such integrals are reviewed in Ref. [11]. For the unimproved lattice theory (8), for example [16],

$$
\Delta\left\langle\phi^{2}\right\rangle_{\mathrm{c}}=\lim _{a_{\text {lat }} \rightarrow 0}\left[\left\langle\phi^{2}\right\rangle_{\text {lat }}-\frac{\Sigma_{\mathrm{U}}}{2 \pi a_{\text {lat }}}\right],
$$

where $\Sigma_{\mathrm{U}}=3.175911535625 \cdots$

There are two limits that must be taken of lattice Monte Carlo data: the continuum limit $u a_{\text {lat }} \rightarrow 0$ and the infinite volume limit $L u \rightarrow \infty$. Fig. 2 shows the dependence of our data on system size $(L u)$ at $u a_{\text {lat }}=6$. Fig. 3 shows the dependence on lattice spacing $\left(u a_{\text {lat }}\right)$ at $L u=576$. From these two figures, it is reasonably clear that our raw data includes reasonably large volumes and reasonably small lattice spacings. We will discuss extrapolations of the infinite-volume continuum limit using finite volume scaling, the known critical exponents of this model, and an analysis of finite lattice-spacing errors.

First, however, we wish to show that one can obtain a quick estimate from the raw data without relying on anything fancy. Table II shows values associated with a selected subset of the largest $L u$ and smallest $u a_{\text {lat }}$ data points from the figures. We take the $\left(L u, u a_{\text {lat }}\right)=$ 


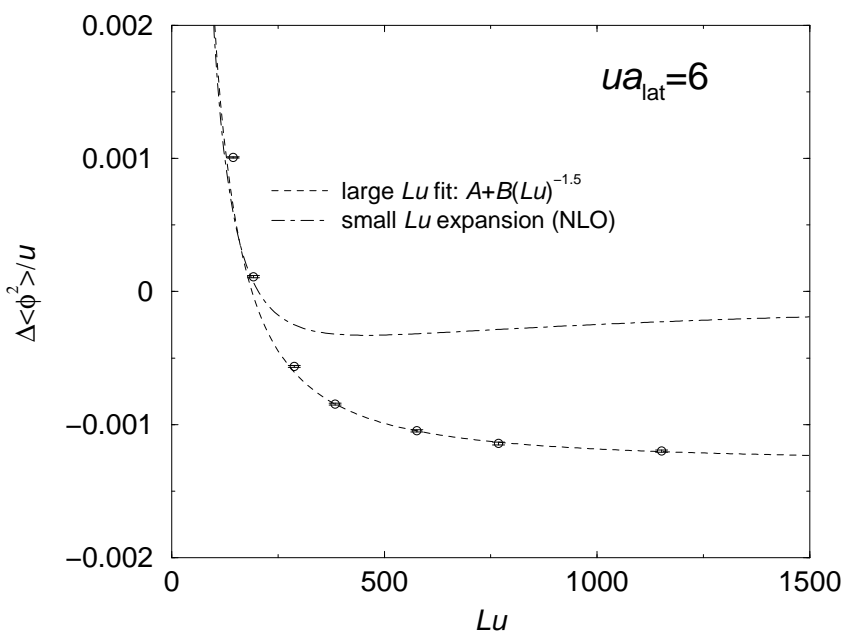

FIG. 2. $O\left(u a_{\text {lat }}\right)$-corrected results for $\Delta\left\langle\phi^{2}\right\rangle_{\mathrm{c}}$ vs. system size at $u a_{\text {lat }}=6$. A numerical fit to large $L$ scaling behavior is shown, which fits the last 4 points with confidence level $61 \%$. Also shown, for comparison, is a small $L u$ expansion 11 of the exact continuum result in finite volume (at next-to-leading order in $L u$ ).

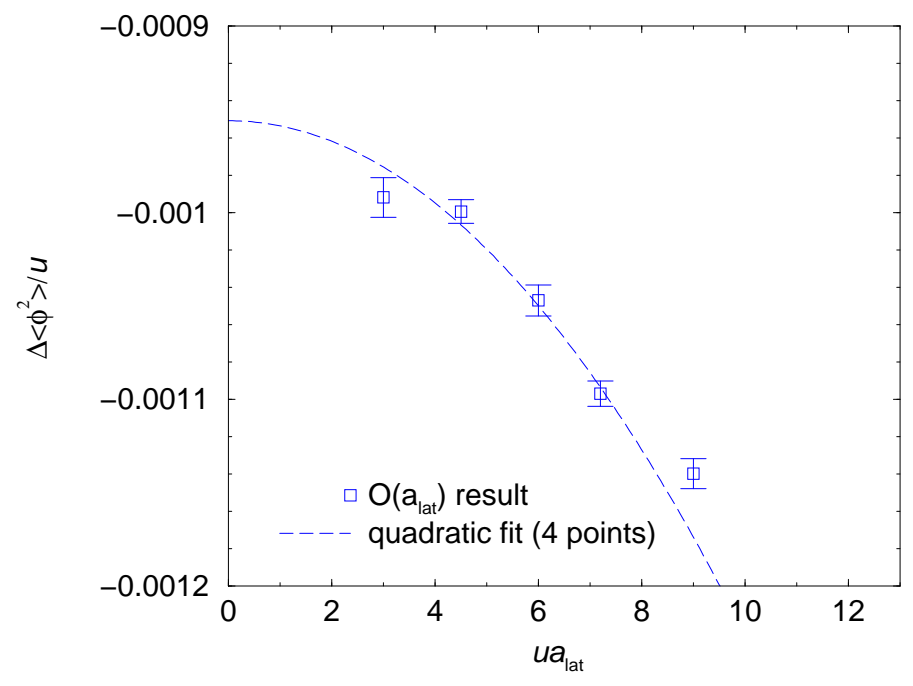

FIG. 3. Results for $\Delta\left\langle\phi^{2}\right\rangle_{\mathrm{c}}$ vs. $u a_{\text {lat }}$ at $L u=576$ that incorporate perturbatively calculated $O\left(u a_{\text {lat }}\right)$ corrections. The line is a fit of $A+B\left(u a_{\text {lat }}\right)^{2}$ to all but the rightmost data point and has confidence level $14 \%$.

\begin{tabular}{|c|c|}
\hline$\left(L u, u a_{\text {lat }}\right)$ & $\Delta\left\langle\phi^{2}\right\rangle_{\mathrm{c}} / u$ \\
\hline$(576,6)$ & $-0.001047(8)$ \\
$(576,3)$ & $-0.000992(11)$ \\
$(1152,6)$ & $-0.001200(9)$ \\
\hline
\end{tabular}

TABLE I. Selected data for $\Delta\left\langle\phi^{2}\right\rangle_{\mathrm{c}} / u$.
$(576,6)$ result as a starting point for our estimate. The finite-volume correction is at least as big as the difference with the value at $(1152,6)$ [which corresponds to doubling $L u$ ] but is unlikely to be double this difference. This difference is roughly -0.00015 (ignoring the small statistical errors), so we might estimate the finite-volume correction to the $L u=576$ value to be somewhere between -0.00015 and -0.00030 . From a similar comparison of the $(576,6)$ and $(576,3)$ data, we might estimate the finite lattice spacing correction to $(576,6)$ to be between roughly +0.00005 and +0.00011 . Adding our corrections and the original $(576,6)$ data point, the final rough estimate of the continuum infinite-volume value would be

$$
\frac{\Delta\left\langle\phi^{2}\right\rangle_{\mathrm{c}}}{u} \simeq-0.00119 \pm 0.00011,
$$

which, by (7) would translate to $c=1.31 \pm 0.12$.

We now summarize a more careful analysis of corrections, detailed in ref. [11]. Our strategy is to start again with $L u=576$ data, extrapolate the continuum limit, and estimate the finite volume correction. To improve the approach to the continuum limit, we have analytically calculated the $O\left(u a_{\text {lat }}\right)$ corrections to $\Delta\left\langle\phi^{2}\right\rangle_{\mathrm{c}}$ and the relation between lattice and continuum values of $u$, using lattice perturbation theory [11]. Fig. 1 shows $L u=144$ data which clearly demonstrates our control of lattice spacing errors. The uncorrected data clearly has a linear dependence on $u a_{\text {lat }}$. But the corrected data fits, to high confidence level, the assertion that the remaining error scales as $\left(u a_{\text {lat }}\right)^{2}$. Based on a similar fit to the data of Fig. 3, we estimate the $u a_{\text {lat }} \rightarrow 0$ result at $L u=576$ as

$$
\left[\frac{\Delta\left\langle\phi^{2}\right\rangle_{\mathrm{c}}}{u}\right]_{L u=576}=-0.000957 \pm 0.000015 .
$$

Finite scaling arguments predict that the large $L u$ corrections to $\Delta\left\langle\phi^{2}\right\rangle_{\mathrm{c}}$ should scale as [11]

$$
\Delta\left\langle\phi^{2}\right\rangle_{\mathrm{c}} \sim A+B L^{-(1-\alpha) / \nu}
$$

if the method of Binder cumulants is used to determine the transition point in finite volume. Here $\alpha \simeq-0.01$ and $\nu=(2-\alpha) / 3 \simeq 0.67$ are the specific heat and correlation length critical exponents of the $\mathrm{O}(2)$ model [17]. Further discussions of fits, and an analysis of corrections to scaling, may be found in ref. [11]. On the basis of these fits, we estimate the finite size correction at $L u=576$ to be $0.000241 \pm 0.000007$. Putting this together with the $L u=576$ continuum extrapolation (12), we obtain the final infinite-volume continuum result

$$
\frac{\Delta\left\langle\phi^{2}\right\rangle_{\mathrm{c}}}{u}=-0.001198 \pm 0.000017
$$

Using Eq. (7) for the weak-interaction limit of $\Delta T_{\mathrm{c}}$, the result for $\Delta\left\langle\phi^{2}\right\rangle_{\mathrm{c}}$ translates to

$$
\frac{\Delta T_{\mathrm{c}}}{T_{0}} \rightarrow(1.32 \pm 0.02) a_{\mathrm{sc}} n^{1 / 3} .
$$




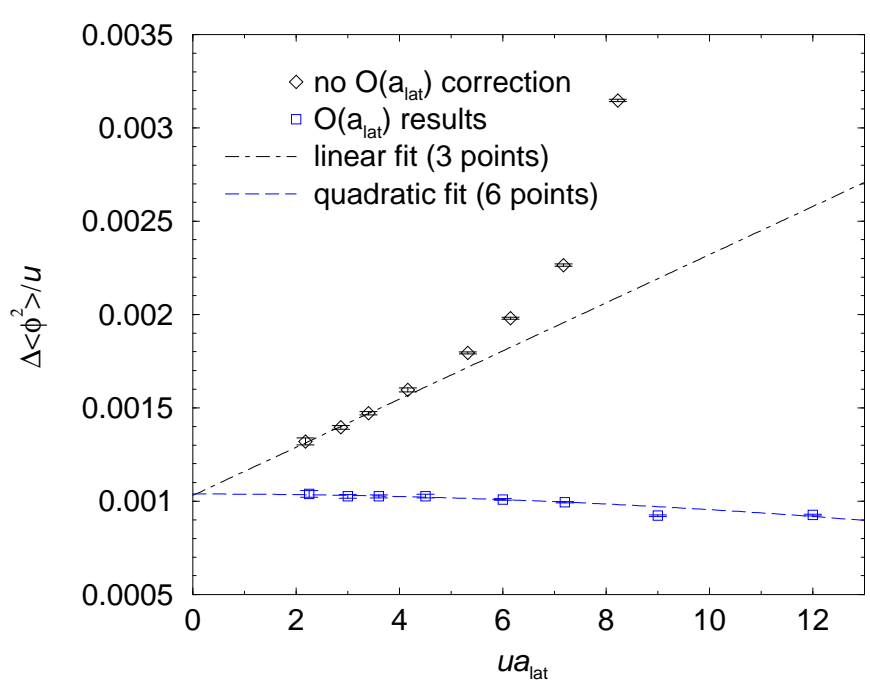

FIG. 4. The squares show $\Delta\left\langle\phi^{2}\right\rangle_{\mathrm{c}}$ vs. $u a_{\text {lat }}$ at $L u=144$ incorporating $O\left(u a_{\text {lat }}\right)$ corrections. The line through them is a fit of the first 6 points to $A+B\left(u a_{\text {lat }}\right)^{2}$ and has confidence level $94 \%$. The diamonds represent the corresponding uncorrected data, with a straight line fit to the first 3 points to guide the eye.

It is interesting to compare our numerical results with results from the large $N$ expansion, depicted by triangles in Fig. 田. In large $N$, one generalizes the $\mathrm{O}(2)$ effective field theory to an $\mathrm{O}(N)$ theory of $N$ real fields, calculates results in powers of $1 / N$, and hopes the expansion will be useful for the case of interest, $N=2$. The leading-order (LO) result $c \simeq 2.33$ [7] is off by $77 \%$, but the next-toleading-order $(\mathrm{NLO})$ result $c \simeq 1.71$ [5] moves in the right direction and is off by only $30 \%$. This is surprisingly good for an expansion that treats $N=2$ as large.

We should comment on the discrepancy of our results with previous numerical calculations in the literature [3.6, shown in Fig. 1, which used a radically different starting point. Rather than using field theory methods and the grand canonical ensemble, they start with the path integral for a large, fixed number of particles in a box. The recent work of Holzmann and Krauth [6], however, makes a flawed assumption at the very beginning: they assume that the integrand of the path integral can be expanded perturbatively in the interaction, and keep only the leading term. This is wrong because interactions cannot, generically, be treated perturbatively at a second-order phase transition.

We believe one likely problem with the older simulations of Grüter et al. is inadequate system size [11. Reppy et al. [18] have reported $c=5.1 \pm 0.9$ from experimental data on He-Vycor systems, but cautions about the data's interpretation may be found in Ref. [5].

As we completed this work, another paper appeared [19] which uses techniques very similar to ours and ob- tains the statistically compatible result $c=1.29 \pm 0.05$.

This work was supported by the U.S. Department of Energy under Grant Nos. DE-FG03-96ER40956 and DEFG02-97ER41027.

[1] G. Baym, J.-P. Blaizot, M. Holzmann, F. Laloë, and D. Vautherin, Phys. Rev. Lett. 83, 1703 (1999).

[2] M. Wilkens, F. Illuminati, and M. Krämer, J. Phys. B 33, L779 (2000).

[3] P. Grüter, D. Ceperley, and F. Laloë, Phys. Rev. Lett. 79, 3549 (1997).

[4] M. Holzmann, P. Grüter, and F. Laloë, Euro. Phys. J. B 10, 739 (1999).

[5] P. Arnold and B. Tomášik, Phys. Rev. A62, 063604 (2000).

[6] M. Holzmann and W. Krauth, Phys. Rev. Lett. 83, 2687 (1999).

[7] G. Baym, J.-P. Blaizot, and J. Zinn-Justin, Europhys. Lett. 49, 150 (2000).

[8] F. de Souza Cruz, M. Pinto, and R. Ramos, condmat/0007151 (unpublished).

[9] H.T.C. Stoof, Phys. Rev. A 45, 8398 (1992); M. Bijlsma and H.T.C. Stoof, Phys. Rev. A 54, 5085 (1996).

[10] F. Dalfovo, S. Giorgine, L. Pitaevski, and S. Stringari, Rev. Mod. Phys. 71, 463 (1999).

[11] P. Arnold and G. Moore, cond-mat/0103227.

[12] J. Goodman and A. D. Sokal, Phys. Rev. Lett. 56, 1015 (1986); J. Goodman and A. D. Sokal, Phys. Rev. D 40, 2035 (1989).

[13] K. Binder, Phys. Rev. Lett. 47, 693 (1981).

[14] The relationship between $r_{\text {lat }}$ and $r_{\text {eff }}$ is derived in Ref. [1], which uses this relation to give a lattice simulation result for $r_{\text {eff }}$. The relationship between $r_{\text {eff }}$ and $\mu$ is derived in Ref. 15, which gives a result for $\mu$ at the transition and uses this to calculate the second-order correction to $T_{\mathrm{c}}$ for a Bose gas in a harmonic trapping potential.

[15] P. Arnold and B. Tomášik, cond-mat/0105147.

[16] G. Watson, Quart. J. Math. (Oxford, 1st series) 10, 266 (1939).

[17] R. Guida and J. Zinn-Justin, J. Phys. A: Math. Gen. 31, 8103 (1998).

[18] J. Reppy, B. Crooker, B. Hebral, A. Corwin, J. He, and G. Zassenhaus, Phys. Rev. Lett. 84, 2060 (2000).

[19] V.A. Kashurnikov, N. Prokof'ev, and B. Svistunov, condmat/0103149; N. Prokof'ev, and B. Svistunov, condmat/0103146 